\title{
Serum liver enzymes serve as prognostic factors in patients with intrahepatic cholangiocarcinoma
}

\author{
This article was published in the following Dove Press journal: \\ OncoTargets and Therapy \\ 6 March 2017 \\ Number of times this article has been viewed
}

\author{
Chenyue Zhang ${ }^{1,2}$ \\ Haiyong Wang ${ }^{1,3}$ \\ Zhouyu Ning ${ }^{1,2}$ \\ Litao Xu $\mathbf{u}^{1,2}$ \\ Liping Zhuang ${ }^{1,2}$ \\ Peng Wang ${ }^{1,2}$ \\ Zhiqiang Meng ${ }^{1,2}$
}

'Department of Integrative Oncology, Fudan University Shanghai Cancer Center, Shanghai, People's Republic of China; ${ }^{2}$ Department of Oncology, Shanghai Medical College, Fudan University, Shanghai, People's Republic of China; ${ }^{3}$ Department of Radiation Oncology, Shandong Cancer Hospita \& Institute, Jinan, People's Republic of China
Correspondence: Zhiqiang Meng; Peng Wang

Department of Integrative Oncology, Fudan University Shanghai Cancer Center, 270 Dong An Road, Shanghai 200032, People's Republic of China

Tel +862164175590 Ext 3628

Fax +86 2I 64437657

Email mengzhq@yeah.net; wangp4I3@I63.com
Objective: Liver functions, reflective of the overall status of the host, have been reported to be important factors affecting the prognosis in many types of cancers. In this study, we explored the influences of liver enzymes albumin (ALB), globulin (GELO), total protein (TP), alkaline phosphatase (ALP), alanine aminotransferase (ALT), aspartate aminotransferase (AST), total bilirubin (TBIL), direct bilirubin (DBIL), gamma glutamyltranspeptidase (GGT), and lactate dehydrogenase (LDH) on the overall survival (OS) in a number of 173 patients with intrahepatic cholangiocarcinoma (ICC).

Patients and methods: Between 2011 and 2015, we enrolled patients with pathologically proven locally advanced or metastatic ICC. The impact of ALB, GELO, TP, ALP, ALT, AST, TBIL, DBIL, GGT, and LDH on OS were analyzed using Kaplan-Meier analysis. Next, the associations between these liver enzymes and OS were evaluated by univariate and multivariate analyses. Finally, the role of these enzymes in OS was evaluated in the subgroups.

Results: Elevated liver enzymes were linked with OS. We revealed that independent prognostic factors of poor outcome were ALP, TBIL, DBIL, and GGT, whereas ALB is a protective factor in ICC patients.

Conclusion: Our results demonstrate that these liver enzymes may serve as valuable predictive markers in ICC patients.

Keywords: liver enzymes, prognosis, overall survival, intrahepatic cholangiocarcinoma

\section{Introduction}

Intrahepatic cholangiocarcinoma (ICC), as one of the most devastating malignancies, comprises $15 \%$ of all primary liver cancers. ${ }^{1,2}$ It is very sinister and signifies a dismal prognosis. Despite being a rare malignancy, studies have shown that a growing number of people are diagnosed with and dying of the disease. ${ }^{3-6}$ Unlike other tumors originating from the liver and gastrointestinal tract, the pathogenesis of ICC remains sophisticated and obscure, thus making the disease more intractable and challenging. ${ }^{7}$ Moreover, the median survival of ICC patients is no more than 12 months, even after different treatments such as surgery, systemic chemotherapy, or radiotherapy. ${ }^{8}$ However, clinical data have shown that survival status varies significantly among patients with ICC. The commonly acknowledged factors that may serve as predictors for survival include intrahepatic satellite lesions, lymph node invasion, and distant metastasis. ${ }^{9-11}$

Cancer is a disease implicating the metabolism of the whole body rather than targeting organs. ${ }^{6}$ Liver functions, indicative of the degree of liver damage, partly reflect the tumor biology status. In clinical practice, we have found that patients with deteriorated liver functions often have unsatisfactory prognosis compared to those with tolerable parameters. 
To date, studies have shown that abnormal expressions of liver enzymes often lead to poor prognosis in a multitude of cancers. Likewise, other liver function markers such as alkaline phosphatase (ALP) and gamma-glutamyltranspeptidase (GGT) were often risk factors in patients with some types of cancers. ${ }^{12-14}$ In addition, alanine aminotransferase (ALT), aspartate aminotransferase (AST), total bilirubin (TBIL), direct bilirubin (DBIL), and lactate dehydrogenase (LDH) were also reported to influence prognosis in some types of cancer. ${ }^{15-18}$

Therefore, in this study we have incorporated a whole set of liver function parameters including albumin (ALB), globulin (GELO), total protein (TP), ALP, ALT, AST, TBIL, DBIL, GGT, and LDH to evaluate their significance in ICC.

\section{Methods}

\section{Ethics statement}

We have gained permission to collect data of the ICC patients from Fudan University Shanghai Cancer Center. This study was approved by the Ethics Committee of the Fudan University Shanghai Cancer Center, Shanghai, People's Republic of China. (This was approved on the December 24, 2012 by the Institutional Review Board and the approved number is 050432-4-1212B). This research was also performed according to the guidelines of the Ethics Committee of Fudan University Shanghai Cancer Center, Shanghai, People's Republic of China. All subjects provided written informed consent.

\section{ICC patients}

From October 2011 to October 2015, 173 patients diagnosed with pathologically proven ICC at the Department of Integrative Oncology, Fudan University, Shanghai Cancer Center were enrolled in this study. A total of 107 (61.8\%) patients were male, and 66 (38.2\%) were female. Among all patients, $36(20.8 \%)$ patients had a diagnosis of locally advanced cancer, while the remaining patients were all at the metastatic stage. Locally advanced ICC refers to unresectable ICC with the tumors invading vascular structures or surrounding organs but confined to the liver and/or regional lymph nodes. For ICC, disease spread to the celiac and/or periaortic and caval lymph nodes is considered distant metastases. The number of patients who had CA19-9 of $1,000 \mathrm{IU} / \mathrm{mL}$ or greater is 64 , accounting for $37.0 \%$ of all the patients. And among all the patients, 99 patients underwent gemcitabinebased chemotherapy, while the remaining 74 patients were subjected to 5-fluorouracil-based chemotherapy. The 5-fluorouracil-based chemotherapy referred to 5-fluorouracil, capecitabine, or S1 (combined tegafur, gimeracil, and oteracil). Gemcitabine-based chemotherapy referred to gemcitabine monotherapy (gemcitabine $1,000 \mathrm{mg} / \mathrm{m}^{2}$ weekly for 7 weeks and, after a 1-week rest, on days 1,8 , and 15 every 4 weeks), or gemcitabine-based combination chemotherapy, including GEMOX (gemcitabine $1,000 \mathrm{mg} / \mathrm{m}^{2}$ on day 1 plus oxaliplatin $100 \mathrm{mg} / \mathrm{m}^{2}$ on day 2 every 14 days) or GP (gemcitabine $1,000 \mathrm{mg} / \mathrm{m}^{2}$ plus cisplatin $25 \mathrm{mg} / \mathrm{m}^{2}$ on days 1,8 , and 15 every 4 weeks).

\section{Measurements of serum liver enzymes}

Blood samples were withdrawn for the detection of the following liver function parameters: ALB, GELO, TP, ALP, ALT, AST, TBIL, DBIL, GGT, and LDH. All these tests were performed prior to all the treatments of these patients. Besides, the levels of serum liver enzymes were measured after two cycles of chemotherapy. The median values of these liver enzymes were chosen as the cutoffs.

\section{Statistical analyses}

Values were expressed as mean with standard deviation. Comparisons between the clinical variables were made using chi-square tests. To compare cumulative survival between patients in different groups, we have used KaplanMeier analysis. And to estimate the difference in survival, we have adopted log-rank test. The associations with survival were evaluated adopting Cox proportional hazards. Multivariate analysis was also conducted using the Cox proportional hazards regression model adjusted for CA19-9. A $P$-value below 0.05 was viewed as reaching the statistical significance. All the analyses were performed using SPSS 17.0 software.

\section{Results Clinicopathological features of ICC patients}

In total, 173 patients with ICC were included in this study. Among them, 107 (61.8\%) were male, the remaining $66(38.2 \%)$ were female. According to American Joint Committee on Cancer staging, a total of $36(20.8 \%)$ patients were diagnosed with locally advanced ICC, while the other patients were all diagnosed with metastatic ICC. Among these patients with metastatic ICC, 53 had celiac, periaortic, and caval lymph node metastases and 48 had intrahepatic metastases, and the rest of them had lung and bone metastases. Among the 173 ICC patients, a total of 99 patients underwent gemcitabine-based chemotherapy, while 74 patients were subjected to 5 -fluorouracil-based chemotherapy. The concrete clinicopathological characteristics of these ICC patients are shown in Table 1. 
Table I Clinical information for intrahepatic cholangiocarcinoma patients

\begin{tabular}{|c|c|c|}
\hline Variables & & $N=173$ \\
\hline Age (years) & Mean \pm SD & $58.83 \pm|| .31$ \\
\hline \multirow[t]{2}{*}{ Gender } & Male & 107 (38.2) \\
\hline & Female & $66(61.8)$ \\
\hline \multirow[t]{2}{*}{ Stage } & Locally advanced & $36(20.8)$ \\
\hline & Metastatic & I37 (79.2) \\
\hline \multirow[t]{2}{*}{ CAI9-9 } & $<\mathrm{I}, 000 \mathrm{IU} / \mathrm{mL}$ & $109(63.0)$ \\
\hline & $\geq \mathrm{I}, 000 \mathrm{IU} / \mathrm{mL}$ & $64(37.0)$ \\
\hline ALB level & Median (range) & $40.7(26-50.9)$ \\
\hline GELO level & Median (range) & $29.9(20.9-55)$ \\
\hline TP level & Median (range) & $71.9(53.3-89.3)$ \\
\hline ALP level & Median (range) & $138(5 \mid .4-1,500)$ \\
\hline ALT level & Median (range) & $26.6(5.0-545.30)$ \\
\hline AST level & Median (range) & $32.6(10-366.2)$ \\
\hline TBIL level & Median (range) & $12.2(4.3-42 \mid .3)$ \\
\hline DBIL level & Median (range) & $4.7(0.8-322.8)$ \\
\hline GGT level & Median (range) & II $3($ II-2,064) \\
\hline LDH level & Median (range) & $192(74-833)$ \\
\hline
\end{tabular}

Abbreviations: ALB, albumin; ALP, alkaline phosphatase; TBIL, total bilirubin; DBIL, direct bilirubin; GGT, gamma glutamyltranspeptidase; GELO, globulin; TP, total protein; ALT, alanine aminotransferase; AST, aspartate aminotransferase; LDH, lactate dehydrogenase; SD, standard deviation.

\section{Poor liver function parameters are associated with shorter overall survival (OS) in patients with ICC}

To explore the significance of liver enzyme parameters in patients with ICC, we evaluated the correlation between the respective liver function marker (ALB, GELO, TP, ALP, ALT, AST, TBIL, DBIL, GGT, and LDH) and OS among these ICC patients. Low ALB was demonstrated to be in an intimate relationship with a decreased OS in ICC patients, as shown in the Kaplan-Meier analysis. Patients with lower ALB have OS of 7.6 months, whereas patients with relatively higher ALB have OS of 13.93 months (log-rank =7.451, $P=0.0013$, Figure 1). In addition, we analyzed the role of other liver enzymes in these ICC patients. We have shown that low levels of ALP, TBIL, DBIL, and GGT were all associated with worse OS (Figure 1). In patients with ICC, we have found a significant correlation between elevated liver enzyme levels (ALP, TBIL, DBIL, GGT) and decreased OS. Among them, the median survival of patients with relatively lower ALP was 14.23 months, whereas the figure plummeted to 5.970 months for patients with comparably higher ALP (Figure 1). And the median survival of patients with high TBIL and DBIL were 7.600 months and 5.670 months, respectively, whereas in those with relatively lower TBIL and DBIL, their median survival can be up to 13.70 months, respectively and 14.23 months, respectively. Therefore, we have drawn the conclusion that decreased ALB level and elevated expressions of ALP, TBIL, DBIL, and GGT indicate shorter OS in patients with metastatic ICC.

\section{Liver enzyme levels can predict OS in ICC patients}

To determine the prognostic power of liver enzyme levels for OS in patients with ICC, univariate analyses were performed in the first place. Results have shown that among all the variables, CA19-9, ALB, ALP, TBIL, DBIL, and GGT have an impact on OS (Table 2). In order to exclude the influences of the interactive effect of these liver enzymes, we performed multivariate analyses to ascertain the independent influence of each of them. Multivariate analysis confirmed that ALB (hazard ratio [HR]: $0.741,95 \%$ confidence interval [CI]: 0.521-1.053, $P=0.095$ for OS), ALP (HR: 1.654, 95\% CI: $1.170-2.337, P=0.004$ for OS), TBIL (HR: $1.624,95 \%$ CI: $1.142-2.310, P=0.007$ for OS), DBIL (HR: $1.856,95 \% \mathrm{CI}$ : 1.304-2.642, $P=0.001$ for OS), and GGT (HR: $1.641,95 \%$ CI: 1.160-2.321, $P=0.005$ for OS) serves as independent prognostic predictors for OS in ICC patients (Table 3).

\section{5-Fluorouracil-based chemotherapy had a more direct influence on the serum liver markers than gemcitabine-based chemotherapy}

To evaluate the effect of different chemotherapies on serum liver enzymes, we have recorded their pre- and postchemotherapy levels. The comparisons have been shown in Table 4. Results showed that gemcitabine-based chemotherapy had a more significant impact on serum liver enzymes than 5-fluorouracil-based chemotherapy.

\section{Serum liver enzymes predict survival within subgroups of patients with ICC}

ICC patients were categorized into the locally advanced and metastatic stage, and those with CA19-9 $<1,000 \mathrm{IU} / \mathrm{mL}$ and those with CA19-9 $\geq 1,000 \mathrm{IU} / \mathrm{mL}$. Serum liver enzymes (ALB, GELO, TP, ALP, ALT, AST, TBIL, DBIL, GGT, and LDH) were significantly correlated with OS in each subgroup (Figure 2). It has proven that the serum liver enzymes can serve as predictive markers in patients with locally advanced ICC and metastatic tumor.

\section{Morphological tumor differences did not affect OS in patients with ICC}

We have also classified the 173 ICC patients into three subgroups according to their morphological tumor differences: 1) the mass forming type, 2) the perihilar invasion type, and 3) 

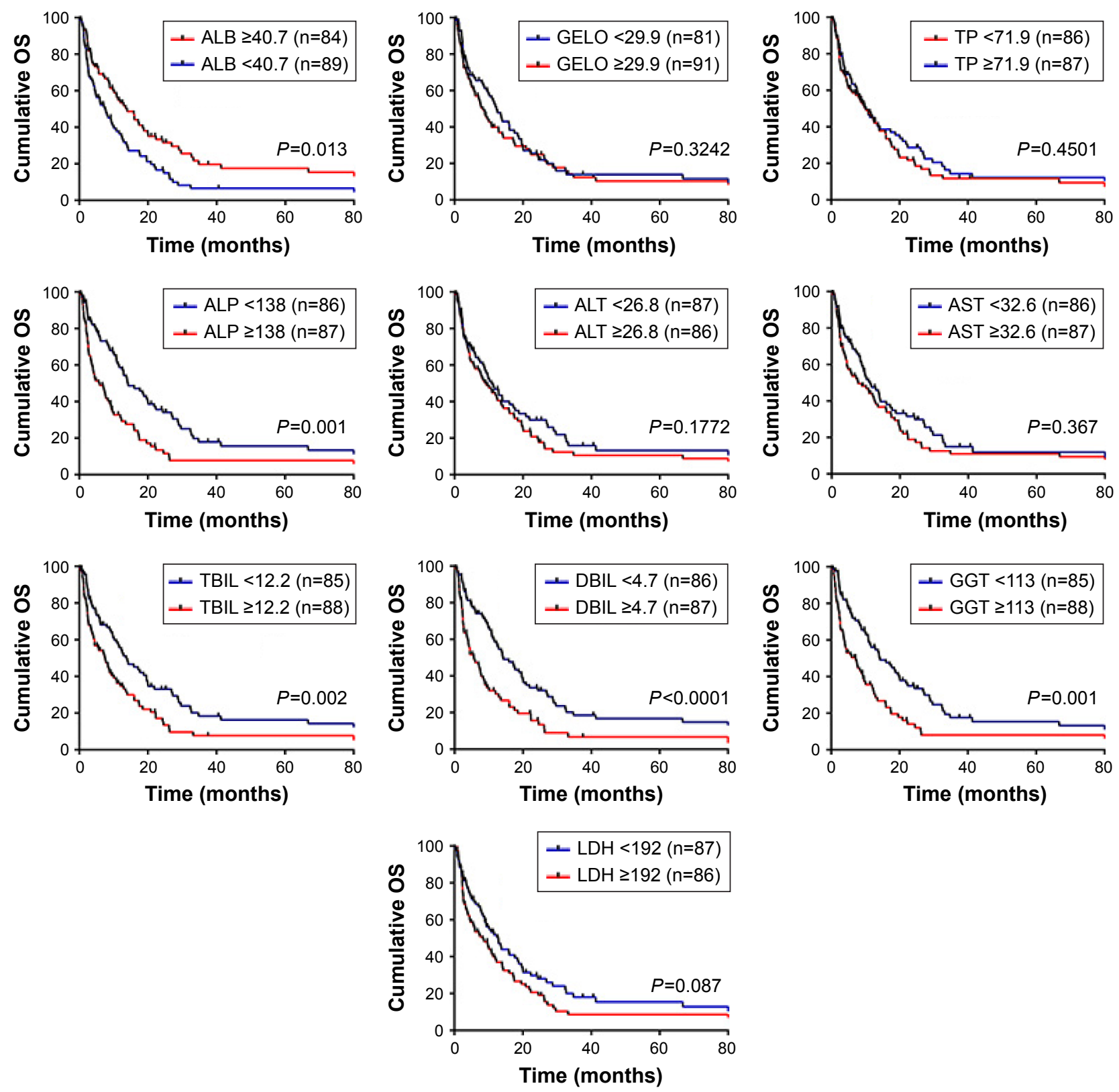

Figure I The associations of serum liver enzymes with OS in patients with ICC.

Abbreviations: ALB, albumin; GELO, globulin; TP, total protein; ALP, alkaline phosphatase; ALT, alanine aminotransferase; AST, aspartate aminotransferase; TBIL, total bilirubin; DBIL, direct bilirubin; GGT, gamma glutamyltranspeptidase; LDH, lactate dehydrogenase; OS, overall survival; ICC, intrahepatic cholangiocarcinoma.

the intraductal growth type. To assess their influences on survival, Kaplan-Meier analysis was performed. It showed that there was no significant statistical difference in OS among the three subtypes (Figure S1). The $P$-values of OS among the three subtypes were 0.1128 (mass forming vs periductal invasion), 0.0739 (periductal invasion vs intraductal growth), and 0.4020 (mass forming vs intraductal growth).

\section{Discussion}

In this study, we have ascertained the prognostic significance of a complete set of liver function parameters in ICC patients. The associations between liver function indices like ALB,
GELO, TP, ALP, ALT, AST, GGT, LDH, TBIL, and DBIL with OS were all analyzed in the cohort of 173 ICC patients in the first place. We have found that a low ALB expression and elevated ALP, TBIL, DBIL, and GGT levels were significantly correlated with shorter OS in patients with ICC. And further univariate and multivariate analyses have found that ALP, TBIL, DBIL, and GGT may serve as independent prognostic predictors for OS in patients with ICC.

In clinical practices, liver functions have long been considered to assume crucial status in the prognosis of many types of cancers such as gallbladder and colorectal cancers. ${ }^{12,19,20}$ Liver functions can be reflected by markers not 
Table 2 Univariate analysis of prognostic factors in intrahepatic cholangiocarcinoma $(\mathrm{N}=173)$

\begin{tabular}{|c|c|c|c|c|c|}
\hline Variables & Subgroup & $\mathbf{N}$ & MST (month) & I-year survival rate (\%) & $P$-value \\
\hline \multirow[t]{2}{*}{ Age (years) } & $<60$ & 79 & 5.97 & 34.18 & 0.3345 \\
\hline & $\geq 60$ & 94 & 8.2 & 35.11 & \\
\hline \multirow[t]{2}{*}{ Gender } & Male & 107 & 7 & 32.71 & 0.807 \\
\hline & Female & 66 & 8.75 & 37.88 & \\
\hline \multirow[t]{2}{*}{ CA19-9 } & $<\mathrm{I}, 000 \mathrm{IU} / \mathrm{mL}$ & 109 & 10.77 & 44.04 & $<0.0001$ \\
\hline & $\geq 1,000 \mathrm{IU} / \mathrm{mL}$ & 64 & 3.8 & 18.75 & \\
\hline \multirow[t]{2}{*}{ ALB } & $<40.7$ & 89 & 6 & 28.57 & 0.013 \\
\hline & $\geq 40.7$ & 84 & 8.75 & 36.73 & \\
\hline \multirow[t]{2}{*}{ GELO } & $<29.9$ & 91 & 9.7 & 40.24 & 0.3242 \\
\hline & $\geq 29.9$ & 81 & 6.77 & 29.67 & \\
\hline \multirow[t]{2}{*}{ TP } & $<71.9$ & 86 & 7.62 & 37.21 & 0.4501 \\
\hline & $\geq 71.9$ & 87 & 7.6 & 32.18 & \\
\hline \multirow[t]{2}{*}{ ALP } & $<138$ & 86 & 11.6 & 48.84 & 0.001 \\
\hline & $\geq 138$ & 87 & 4.47 & 20.69 & \\
\hline \multirow[t]{2}{*}{ ALT } & $<26.6$ & 87 & 7.28 & 33.72 & 0.1772 \\
\hline & $\geq 26.6$ & 86 & 7.76 & 34.48 & \\
\hline \multirow[t]{2}{*}{ AST } & $<32.6$ & 86 & 9.04 & 36.05 & 0.367 \\
\hline & $\geq 32.6$ & 87 & 5.66 & 33.33 & \\
\hline \multirow[t]{2}{*}{ TBIL } & $<12.2$ & 85 & 10.48 & 44.05 & 0.002 \\
\hline & $\geq 12.2$ & 88 & 5.54 & 26.14 & \\
\hline \multirow[t]{2}{*}{ DBIL } & $<4.7$ & 86 & 11.93 & 50 & $<0.0001$ \\
\hline & $\geq 4.7$ & 87 & 4 & 19.54 & \\
\hline \multirow[t]{2}{*}{ GGT } & $<113$ & 85 & 11.86 & 48.24 & 0.001 \\
\hline & $\geq 113$ & 88 & 4.33 & 21.59 & \\
\hline \multirow[t]{2}{*}{ LDH } & $<192$ & 87 & 9.01 & 39.53 & 0.087 \\
\hline & $\geq 192$ & 86 & 5.97 & 29.89 & \\
\hline \multirow[t]{2}{*}{ Chemotherapy } & Gemcitabine-based & 99 & 8.7 & 38.38 & 0.2284 \\
\hline & 5-fluorouracil-based & 74 & 7.5 & 22.22 & \\
\hline
\end{tabular}

Abbreviations: ALB, albumin; ALP, alkaline phosphatase; TBIL, total bilirubin; DBIL, direct bilirubin; GGT, gamma glutamyltranspeptidase; GELO, globulin; TP, total protein; ALT, alanine aminotransferase; AST, aspartate aminotransferase; LDH, lactate dehydrogenase; MST, median survival time.

only like ALB, GELO, and TP, indicators of the nutritional status, but also like ALP, ALT, AST, GGT, LDH, TBIL, and DBIL, indices reflecting liver damage. Thus, we first evaluated the effect of all of these liver function parameters on the OS in ICC patients. We have found that five of them
(ALB, ALP, GGT, TBIL, and DBIL) were significantly associated with OS. Among them, elevated ALB was correlated with better OS, while increased expressions of ALP, GGT, TBIL, and DBIL were linked with shorter OS in patients with ICC.

Table 3 Association of biliary enzyme with OS in patients with intrahepatic cholangiocarcinoma (N=I73)

\begin{tabular}{|c|c|c|c|c|c|c|}
\hline Variables & Group & Case number & HR (95\% Cl) & $P$-value & $\begin{array}{l}\text { Adjusted HR } \\
(95 \% \mathrm{Cl})\end{array}$ & $\begin{array}{l}\text { Adjusted } \\
P \text {-value }\end{array}$ \\
\hline ALB & $<40.7 / \geq 40.7$ & $84 / 89$ & $\begin{array}{l}0.654 \\
(0.466-0.917)\end{array}$ & 0.014 & $\begin{array}{l}0.74 \mathrm{I} \\
(0.52 \mathrm{I}-\mathrm{I} .053)\end{array}$ & 0.095 \\
\hline ALP & $<|38| \geq \mid 38$ & $86 / 87$ & $\begin{array}{l}1.818 \\
(1.290-2.560)\end{array}$ & 0.001 & $\begin{array}{l}1.654 \\
(1.170-2.337)\end{array}$ & 0.004 \\
\hline TBIL & $<|2.2| \geq \mid 2.2$ & $85 / 88$ & $\begin{array}{l}\text { I.692 } \\
(1.198-2.389)\end{array}$ & 0.003 & $\begin{array}{l}1.624 \\
(1.142-2.310)\end{array}$ & 0.007 \\
\hline DBIL & $<4.7 / \geq 4.7$ & $86 / 87$ & $\begin{array}{l}1.984 \\
(1.403-2.804)\end{array}$ & $<0.000 \mathrm{I}$ & $\begin{array}{l}1.856 \\
(1.304-2.642)\end{array}$ & 0.001 \\
\hline GGT & $<113 / \geq 113$ & $85 / 88$ & $\begin{array}{l}\mathrm{I} .804 \\
(\mathrm{I} .28 \mathrm{I}-2.540)\end{array}$ & 0.001 & $\begin{array}{l}1.641 \\
(1.160-2.321)\end{array}$ & 0.005 \\
\hline Chemotherapy & $\begin{array}{l}\text { 5-fluorouracil-based/ } \\
\text { gemcitabine-based }\end{array}$ & $74 / 99$ & $\begin{array}{l}1.277 \\
(0.902-1.808)\end{array}$ & 0.148 & $\begin{array}{l}1.290 \\
(0.914-1.821)\end{array}$ & 0.168 \\
\hline
\end{tabular}

Note: Analysis was adjusted for CA19-9 ( $<1,000$ vs $\geq 1,000)$ variables identified by multivariate Cox regression analyses.

Abbreviations: OS, overall survival; HR, hazard ratio; Cl, confidence interval; ALB, albumin; ALP, alkaline phosphatase; TBIL, total bilirubin; DBIL, direct bilirubin; GGT, gamma glutamyltranspeptidase. 
Table 4 The effect of chemotherapies on the levels of serum liver enzyme markers in patients with intrahepatic cholangiocarcinoma $(\mathrm{N}=173)$

\begin{tabular}{|c|c|c|c|c|c|c|c|c|c|c|c|c|c|c|c|}
\hline \multirow[t]{2}{*}{ Chemotherapy } & \multicolumn{3}{|l|}{ TBIL } & \multicolumn{3}{|l|}{ DBIL } & \multicolumn{3}{|l|}{ ALP } & \multicolumn{3}{|l|}{ GGT } & \multicolumn{3}{|l|}{ ALB } \\
\hline & Prior & Post & $P$-value & Prior & Post & $P$-value & Prior & Post & $P$-value & Prior & Post & $P$-value & prior & post & $P$-value \\
\hline Gem & 12.2 & 17.8 & 0.0346 & 4.8 & 8 & 0.0297 & 137.9 & 169.1 & 0.0008 & 117 & 159 & 0.1487 & 40 & 36.3 & $<0.0001$ \\
\hline $5 \mathrm{Fu}$ & 12.3 & 15.3 & 0.6423 & 4.6 & 6.7 & 0.3848 & 138 & 160.3 & 0.4132 & 109.5 & 125.5 & 0.1245 & $4 I .1$ & 36.75 & $<0.000$ I \\
\hline
\end{tabular}

Abbreviations: Gem, gemcitabine; 5Fu, 5-fluorouracil; TBIL, total bilirubin; DBIL, direct bilirubin; ALP, alkaline phosphatase; GGT, gamma glutamyltranspeptidase; ALB, albumin.

The liver enzyme levels tested in clinical practice can be categorized into two categories: 1) hepatocellular predominance characterized by increased ALT and AST levels, and 2) cholestatic predominance featured by elevated ALP and GGT..$^{21,22}$ In our present study, associations between increased
ALP, GGT, and poorer OS were found, which can be indicative of the cholestatic dysfunction among the ICC patients. Besides, ALT and AST levels were tested to examine their associations with OS among these ICC patients. However, we did not observe significant correlations between them.
ALB


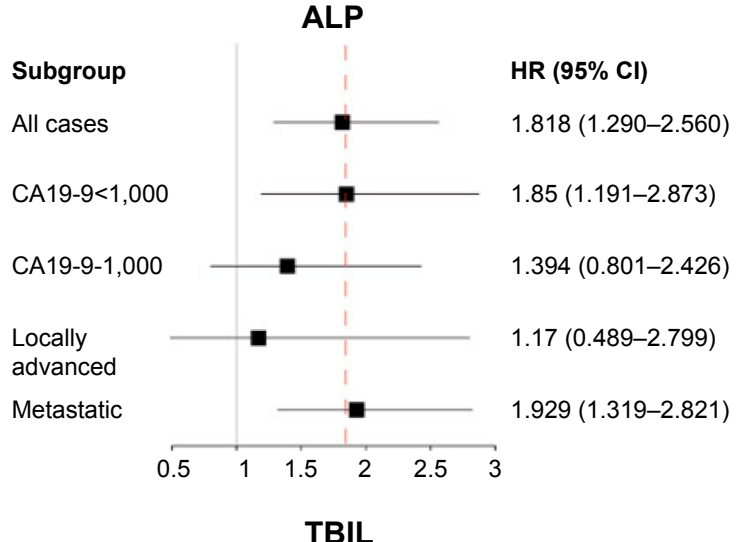

Subgroup

All cases

CA19-9<1,000

CA19-9-1,000

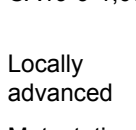

Metastatic
HR $(95 \% \mathrm{Cl})$

$1.692(1.198-2.389)$

$1.662(1.064-2.597)$

$1.655(0.950-2.885)$

$1.52(0.601-3.846)$

$1.665(1.135-2.442)$



Figure 2 Hazard ratios (HRs) of the serum liver enzymes were analyzed in the subgroups of patients with intrahepatic cholangiocarcinoma. Abbreviations: ALB, albumin; ALP, alkaline phosphatase; TBIL, total bilirubin; DBIL, direct bilirubin; GGT, gamma glutamyltranspeptidase. 
This can be attributed to the fact that ALT and AST were markers closely related to hepatocellular predominance. The patients we incorporated into the study were all ICC patients, who may have impaired liver function more linked with the cholestatic aspect. ALP and GGT, as two liver enzymes reflecting the cholestatic metabolism, can be at aberrant levels among ICC patients, and their levels can be linked with survival in patients with ICC. Indeed, these two enzymes have long been reported to be critical references in the diagnosis of malignant tumors such as gallbladder cancer and liver cancer. ${ }^{12,23,24}$ However, the values of a complete set of liver enzymes have been little explored in ICC thus far. Most studies have investigated the role of liver enzymes in hepatocellular carcinoma, the most commonly seen hepatic carcinoma. However, only few studies have expanded the values of liver enzymes in ICC. Only some studies reported the potential role of a few liver enzymes in ICC. To the best of our knowledge, this is one of the few studies that explored the whole set of liver enzymes in ICC.

Among all the liver enzymes, GGT and ALP have always been given due attention as the predicative markers in cancer patients. GGT has been reported to be the key liver enzyme regulating glutathione metabolism. ${ }^{25-27}$ An increased GGT level has been considered as an alarming indicator of liver disease by medical practitioners. Recent studies also found abnormal GGT levels in some types of tumor. The prognostic significance of GGT in esophageal squamous cell carcinoma, gallbladder cancer, hepatocellular carcinoma, human metastatic colorectal cancer, and renal cell carcinoma has been reported. ${ }^{12,24,28}$ A recent study has highlighted the predicative role of increased GGT as a marker suggesting aggressive tumor behavior in patients with ICC. ${ }^{29}$ In the present study, we also confirmed the unfavorable prognosis of elevated GGT in ICC patients, which is in line with the previous study. The underlying mechanisms of the association between GGT and poor prognosis have always been an issue of exploration and investigation. GGT overexpression in cells has been reported to be linked with DNA damage and oxidized bases, involved in the inflammatory process in the extracellular microenvironment. ${ }^{25-27}$ The GGT elevation may be produced from the tumor as a means of self-defense to counteract oxidized wastes. Besides, the reactive oxygen species can be generated by GGT, which may contribute to carcinogenesis via modulating a series of pathways regarding proliferation and apoptosis. ${ }^{30-32}$ Thus, GGT, as a reliable and sensitive indicator of the liver microenvironment, if not all, may serve as a predictor of prognosis in ICC patients.

Besides, serum ALP was also proven to be of value in predicting OS among the ICC patients in the present study.
ALP existing in the epithelia of the bile duct is involved in the transportation of metabolites, which serves as an enzyme regulating the metabolic event. Studies reported that cholestasis may promote ALP secretion from the cell membrane. ${ }^{33}$ Since most ICC patients may be subject to cholestasis, it is not surprising that patients with ICC may have elevated ALP levels. In this study, we found that the degree of ALP expression is indicative of the survival status in the cohort of ICC patients. Since mere ALP elevation can be reflective of many diseases other than liver diseases, such as bone diseases, it entails the necessity to combine some enzymes to authentically evaluate the prognosis of ICC patients. GGT and ALP are often evaluated together to reflect the liver status in patients, thus their consistent elevation may be suggestive of a deteriorated liver injury, which signifies a dismal prognosis. Additionally, other liver enzymes found to be of prognostic significance were TBIL and DBIL. The OS of ICC patients with lower TBIL was significantly longer than those with higher TBIL. Similarly, a lower DBIL expression is also linked with a longer OS. However, for ALT and AST, they have shown a modest impact on the OS of the ICC patients, which can be partially attributed to the relatively smaller number of ICC patients. Although their associations with OS were statistically insignificant, their values cannot be ignored in evaluating the survival of ICC patients. As we performed univariate analyses, ALB, ALP, TBIL, DBIL, and GGT have statistical significance in OS in these ICC patients. To authentically ascertain the independent significance of each liver enzyme, multivariate Cox regression analyses have also been performed to confirm their respective link with OS. Besides, CA19-9 was adjusted to rule out the influence of CA19-9 on these liver enzymes in the multivariate Cox regression analyses.

Lastly, the prognostic values of these serum liver enzymes (ALB, ALP, TBIL, DBIL, and GGT) in predicting OS in the subgroups of patients with ICC have also been verified.

Our present study has some strengths. This is one of the few studies that have incorporated almost all of the liver enzymes to investigate their potential values as predicative markers among ICC patients. Previous studies either investigated the enzymes in tumors other than ICC or only explored a few of them in ICC. However, some shortcomings also exist. 1) Our study only entails a relatively small amount of ICC patients due to the small proportion of patients diagnosed with ICC compared with hepatocellular carcinoma (HCC). It would have provided a stronger validation of our findings if a larger sample was provided. 2) Since the serum expressions of some liver enzymes could be affected by some medications, it is necessary to have the detailed data of clinical variables of each patient regarding smoking, drinking, and medication. 3) Since the ICC patients 
we recruited at our department are those with locally advanced and metastatic ICC, we regret not being able to recruit those at earlier stages. It is more important to find patients with these markers earlier in order to provide better treatment, which would make more sense in clinical practice. However, these patients are incompletely recorded in our study, which may hinder further analyses of their associations.

In conclusion, our present study provided the first evidences that elevated liver enzymes like ALP, TBIL, DBIL, and GGT indicate poor OS in ICC patients, while ALB suggests better prognosis. Therefore, these liver enzymes may serve as valuable predicative markers in ICC patients.

\section{Acknowledgment}

This study was supported by Natural Science Foundation of China (No 81273954) and Shanghai Health Bureau (XBR2013083).

\section{Disclosure}

The authors report no conflicts of interest in this work.

\section{References}

1. Patel T. Cholangiocarcinoma - controversies and challenges. Nat Rev Gastroenterol Hepatol. 2011;8(4):189-200.

2. Patel T. Worldwide trends in mortality from biliary tract malignancies. BMC Cancer. 2002;2:10.

3. Braconi C, Patel T. Cholangiocarcinoma: new insights into disease pathogenesis and biology. Infect Dis Clin North Am. 2010;24(4):871-884.

4. Chu KM, Lai EC, Al-Hadeedi S, et al. Intrahepatic cholangiocarcinoma. World J Surg. 1997;21(3):301-305.

5. Kawarada Y, Yamagiwa K, Das BC. Analysis of the relationships between clinicopathologic factors and survival time in intrahepatic cholangiocarcinoma. Am J Surg. 2002;183(6):679-685.

6. Patel T. Cholangiocarcinoma. Nat Clin Pract Gastroenterol Hepatol. 2006;3(1):33-42.

7. Patel $T$. Increasing incidence and mortality of primary intrahepatic cholangiocarcinoma in the United States. Hepatology. 2001;33(6): 1353-1357.

8. Jaiswal M, LaRusso NF, Burgart LJ, Gores GJ. Inflammatory cytokines induce DNA damage and inhibit DNA repair in cholangiocarcinoma cells by a nitric oxide-dependent mechanism. Cancer Res. 2000; 60(1):184-190.

9. Valle J, Wasan H, Palmer DH, et al. Cisplatin plus gemcitabine versus gemcitabine for biliary tract cancer. $N$ Engl J Med. 2010;362(14): $1273-1281$.

10. Sano T, Shimada K, Sakamoto Y, Ojima H, Esaki M, Kosuge T. Prognosis of perihilar cholangiocarcinoma: hilar bile duct cancer versus intrahepatic cholangiocarcinoma involving the hepatic hilus. Ann Surg Oncol. 2008;15(2):590-599.

11. Li MX, Bi XY, Li ZY, et al. Impaction of surgical margin status on the survival outcome after surgical resection of intrahepatic cholangiocarcinoma: a systematic review and meta-analysis. J Surg Res. 2016;203(1): $163-173$.

12. Xu XS, Miao RC, Zhang LQ, et al. Model Based on Alkaline Phosphatase and Gamma-Glutamyltransferase for Gallbladder Cancer Prognosis. Asian Pac J Cancer Prev. 2015;16(15):6255-6259.

13. Hofbauer SL, Stangl KI, de Martino M, et al. Pretherapeutic gammaglutamyltransferase is an independent prognostic factor for patients with renal cell carcinoma. Br J Cancer. 2014;111(8):1526-1531.
14. Ma H, Zhang L, Tang B, et al. $\gamma$-Glutamyltranspeptidase is a prognostic marker of survival and recurrence in radiofrequency-ablation treatment of hepatocellular carcinoma. Ann Surg Oncol. 2014;21(9): 3084-3089.

15. Gao C, Fang L, Li JT, Zhao HC. Significance and prognostic value of increased serum direct bilirubin level for lymph node metastasis in Chinese rectal cancer patients. World J Gastroenterol. 2016;22(8): 2576-2584.

16. Li N, Xu M, Cai MY, et al. Elevated serum bilirubin levels are associated with improved survival in patients with curatively resected non-smallcell lung cancer. Cancer Epidemiol. 2015;39(5):763-768.

17. Kim WR, Flamm SL, Di Bisceglie AM, Bodenheimer HC; Public Policy Committee of the American Association for the Study of Liver Disease. Serum activity of alanine aminotransferase (ALT) as an indicator of health and disease. Hepatology. 2008;47(4):1363-1370.

18. Faloppi L, Bianconi M, Memeo R, et al. Lactate Dehydrogenase in Hepatocellular Carcinoma: Something Old, Something New. Biomed Res Int. 2016;2016:7196280.

19. Carr BI, Guerra V. A Hepatocellular Carcinoma Aggressiveness Index and Its Relationship to Liver Enzyme Levels. Oncology. 2016;90(4): 215-220.

20. He WZ, Guo GF, Yin CX, et al. Gamma-glutamyl transpeptidase level is a novel adverse prognostic indicator in human metastatic colorectal cancer. Colorectal Dis. 2013;15(8):e443-e452.

21. Wroblewski F. The clinical significance of transaminase activities of serum. Am J Med. 1959;27:911-923.

22. Schlaeger R, Haux D, Kattermann R. Studies on the mechanism of the increase in serum alkaline phosphatase activity in cholestasis: significance of the hepatic bile acid concentration for the leakage of alkaline phosphatase from rat liver. Enzyme. 1982;28(1):3-13.

23. Song P, Inagaki Y, Wang Z, et al. High Levels of Gamma-Glutamyl Transferase and Indocyanine Green Retention Rate at $15 \mathrm{~min}$ as Preoperative Predictors of Tumor Recurrence in Patients With Hepatocellular Carcinoma. Medicine (Baltimore). 2015;94(21):e810.

24. Yao DF, Dong ZZ. Hepatoma-related gamma-glutamyl transferase in laboratory or clinical diagnosis of hepatocellular carcinoma. Hepatobiliary Pancreat Dis Int. 2007;6(1):9-11.

25. Obrador E, Carretero J, Ortega A, et al. Gamma-Glutamyltranspeptidase overexpression increases metastatic growth of B16 melanoma cells in the mouse liver. Hepatology. 2002;35(1):74-81.

26. Russo A, DeGraff W, Friedman N, Mitchell JB. Selective modulation of glutathione levels in human normal versus tumor cells and subsequent differential response to chemotherapy drugs. Cancer Res. 1986; 46(6):2845-2848.

27. Pastore A, Federici G, Bertini E, Piemonte F. Analysis of glutathione: implication in redox and detoxification. Clin Chim Acta. 2003;333(1): 19-39.

28. Yang F, Zhang S, Yang H, et al. Prognostic significance of gammaglutamyltransferase in patients with resectable esophageal squamous cell carcinoma. Dis Esophagus. 2015;28(5):496-504.

29. Yin X, Zheng SS, Zhang BH, et al. Elevation of serum $\gamma$-glutamyltransferase as a predictor of aggressive tumor behaviors and unfavorable prognosis in patients with intrahepatic cholangiocarcinoma: analysis of a large monocenter study. Eur J Gastroenterol Hepatol. 2013; 25(12):1408-1414.

30. Corti A, Duarte TL, Giommarelli C, et al. Membrane gamma-glutamyl transferase activity promotes iron-dependent oxidative DNA damage in melanoma cells. Mutat Res. 2009;669(1-2):112-121.

31. Dominici S, Paolicchi A, Lorenzini E, et al. Gamma-glutamyltransferasedependent prooxidant reactions: a factor in multiple processes. Biofactors. 2003;17(1-4):187-198.

32. Dominici S, Valentini M, Maellaro E, et al. Redox modulation of cell surface protein thiols in U937 lymphoma cells: the role of gammaglutamyl transpeptidase-dependent $\mathrm{H}_{2} \mathrm{O}_{2}$ production and S-thiolation. Free Radic Biol Med. 1999;27(5-6):623-635.

33. Fishman WH. Alkaline phosphatase isoenzymes: recent progress. Clin Biochem. 1990;23(2):99-104. 


\section{Supplementary material}

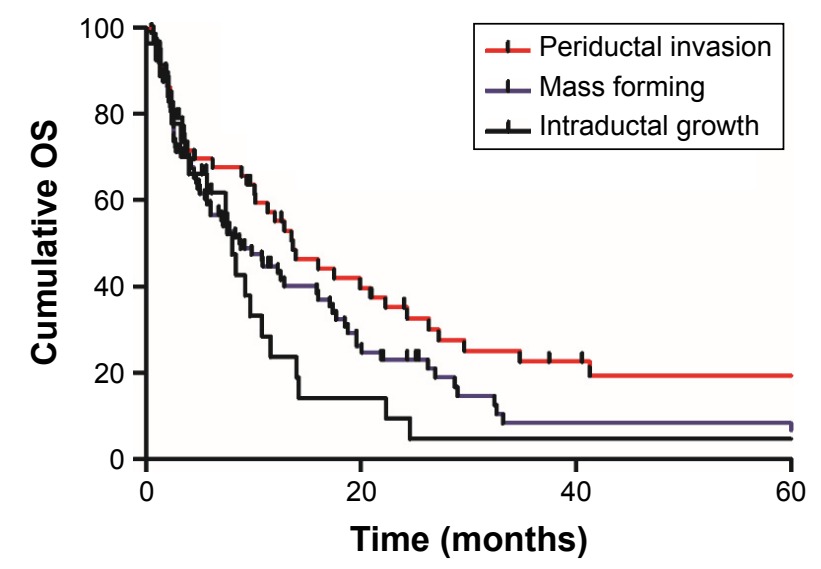

Figure SI The associations between morphological tumor differences and survival were analyzed.

Abbreviation: OS, overall survival.

\section{Publish your work in this journal}

OncoTargets and Therapy is an international, peer-reviewed, open access journal focusing on the pathological basis of all cancers, potential targets for therapy and treatment protocols employed to improve the management of cancer patients. The journal also focuses on the impact of management programs and new therapeutic agents and protocols on patient perspectives such as quality of life, adherence and satisfaction. The manuscript management system is completely online and includes a very quick and fair peer-review system, which is all easy to use. Visit http://www.dovepress.com/testimonials.php to read real quotes from published authors.

\footnotetext{
Submit your manuscript here: http://www.dovepress.com/oncotargets-and-therapy-journal
} 